\title{
Anti-Breast Cancer Activities of 8-Hydroxydaidzein by Targeting Breast Cancer Stem-Like Cells
}

\author{
Sadegh Rajabi ${ }^{1}$, Maryam Shojaee ${ }^{2}$, Ali Malmir ${ }^{3}$, Mostafa Rezaei Tavirani ${ }^{4}$, Shokoofe Noori ${ }^{1}$ \\ ${ }^{1}$ Department of clinical Biochemistry, School of Medicine, Shahid Beheshti University of Medical Sciences, Tehran, Iran. \\ ${ }^{2}$ Department of Biology, Payame Noor University of Mashhad, Mashhad, Iran. ${ }^{3}$ Department of Cellular and Molecular \\ Biology, Faculty of Life Sciences and Biotechnology, Shahid Beheshti University G.C, Tehran, Iran. ${ }^{4}$ Proteomics Research \\ Center, Faculty of Paramedical Sciences, Shahid Beheshti University of Medical Sciences, Tehran, Iran.
}

Received, December 19, 2019; Revised, February 16, 2020; Accepted, March 9, 2020; Published, March 11, 2020.

\begin{abstract}
Purpose: Cancer stem cells (CSCs) play an important role in various stages of cancer development and therapy refractoriness. 8-Hydroxydaidzein (8-OHD) has revealed anti-cancer activity in different tumors. Accordingly, we aimed to assess the effects of 8-OHD on the suppression of breast cancer stem-like cells (BCSCs). Methods: The anti-proliferative and pro-apoptotic properties of 8-OHD were examined by MTS assay and flowcytometry. The expression levels of stemness markers and JAK2/STAT proteins were measured by quantitative real time-PCR (qRT-PCR) and western blotting, respectively. Results: 8-OHD significantly decreased three out of six stemness markers and remarkably reduced viability and induced apoptosis of spheroidal and parental cells compared to controls. Further experiments using CD95L, as a death ligand, and ZB4 antibody, as an extrinsic apoptotic pathway blocker, showed that 8-OHD induced apoptosis through the intrinsic pathway, proposing a mechanism by which 8-OHD triggers apoptosis. Results of western blot analysis also revealed a marked decline in the phosphorylation of JAK2 and STAT proteins. Conclusion: Our study, for the first time, elucidated an anti-BCSC activity for 8-OHD via decreasing stemness markers, inducing toxicity and stimulating apoptosis in these cells and parental cells. Our results also suggested a novel mechanism by which 8-OHD induces apoptosis in BCSCs.
\end{abstract}

\section{INTRODUCTION}

According to the recent World Health Organization (WHO) report, cancer is the second leading cause of mortality globally, resulting in 8.8 million deaths during 2015 (1). Conventional cancer treatments, such as chemotherapy, surgery, and radiotherapy are not effective in curing cancer and they have many side effects as well (2). Therefore, it is necessary to find new therapeutic approaches that completely treat tumors without any side effect. Cancer stem cells (CSCs) play a pivotal role in tumor development, metastasis, recurrence and they are generally resistant to conventional therapies. Therefore, the removal of the cancer stem cell from a tumor could lead to better clinical outcomes (3, $4)$. Breast cancer is the most frequently diagnosed cancer among women and the leading cause of cancer mortality worldwide (5). Breast cancer has been reported as the first tumor from which CSCs were identified and isolated (6). Several putative stemness markers such as Wint 1 , notch $1, \beta$-catenin, CXCR4, SOX2, and ALDH3A1 have been reported for CSCs that they determine the stem cell characteristics of these cells (7-9). Breast CSCs (BCSCs) have been evidenced that show relative resistance to radio- and chemotherapy suggesting the need for the new CSC-targeted therapies for this type of cancer (10). To meet this goal, natural products are the best known candidates for use in treating breast tumors by targeting various signaling molecules and pathways in BCSCs (11). Janus kinase (JAK)/transducer and Activator of Transcription 3 (STAT3) pathway has been revealed to have important roles in the regulation of normal and cancer stem cells behaviors and the inhibition of this pathway could cease tumor growth and induces apoptosis in several cancers (12-14). Natural products as rich sources of novel, bioactive compounds with ability to interact with various cellular targets and with limited painful side-effects have been shown to act as anti-cancer agents by inducing cell death in CSCs, causing CSCs to

Corresponding Author: Shokoofe Noori, Department of Biochemistry, School of Medicine, Shahid Beheshti University of Medical Sciences, Tehran, Iran; E-mail: shnoori85@yahoo.com. 
differentiate, or sensitizing CSCs to conventional chemotherapy treatments (15). Daidzein $\left(4^{\prime}, 7-\right.$ dihydroxyisoflavone) or 7-Hydroxy-3-(4hydroxyphenyl) chromen-4-one is one of the main isoflavonoids found in soybeans that has several pharmacologic properties, such as antioxidant properties and inhibition of tyrosine kinases as well as anticancer and anti-inflammatory properties (16). 8-Hydroxydaidzein (8-OHD) is a hydroxylated derivative of daidzein that can be produced from daidzein, soybean, soybean extract, and soygerm koji by microorganisms isolated from fermented soybean foods or can be converted from daidzein in human and rat liver $(17,18)$. Antioxidative and anti-inflammatory properties of 8-OHD have been reported in previous studies (19-21). 8-OHD also significantly induces apoptosis and reduces mRNA expression levels of multidrug resistance protein 1 (MDR1), MDR-associated protein (MRP) 1, and MRP2 in human colon adenocarcinoma Caco-2 cells, suggesting that the 8-OHD can be involved in intensifying cytotoxicity and preventing resistance to therapy in cancer cells (22). In the current study, we first attempted to enrich breast cancer stem-like cells (BCS-LCs) in spheroidal structures called mammosphere, and then we tried to examine the effects of 8-OHD on the viability, apoptosis and the eradication of stem cell characteristics of BCS-LCs. Our study, for the first time, established an antiBCSC effects for 8-OHD, which were exhibited by reducing stemness markers and proliferation as well as the induction of apoptosis in these cells. Also, we suggested a novel mechanism by which 8-OHD stimulates apoptosis in BCSCs.

\section{METHODS}

\section{Mammosphere Cell Culture and Treatment}

The human breast cancer cell line MCF7 was purchased from Pasteur Institute, Tehran, Iran. The cells were cultured in RPMI1640 containing 10\% FBS and streptomycin/penicillin $(100 \mathrm{U} / \mathrm{ml})$. The cells were incubated at $37^{\circ} \mathrm{C}$ in an atmosphere of $5 \% \mathrm{CO} 2$. Mammosphere structure was formed and cultured according to the method that was described by Lombardo, et al. (23). These spheroidal structures were thus dissociated by Accutase ${ }^{\mathrm{TM}}$ and seeded into 96-well culture plates at a density of 50 cells per well and were cultured by using serumfree media supplemented by epidermal growth factor (EGF) and basic fibroblast growth factor (bFGF) for 7 days. 8-OHD (Sigma-Aldrich, Saint Louis, MO, USA) was dissolved in Dimethyl sulfoxide (DMSO, Sigma-Aldrich, Saint Louis, MO, USA) and the cultured cells were treated with this compound for $48 \mathrm{~h}$. The number of mammospheres in each well was counted under a microscope. The sphere formation rate was determined as the percentage of sphere-forming cells relative to the total number of cells seeded.

\section{Cell Viability and Apoptosis Assay}

Cell viability was assayed using the Cell Titer 96 Aqueous One Solution Cell Proliferation Assay kit [MTS, 3-(4, 5-dimethylthiazol-2 yl)-5-(3carboxymethoxyphenyl)-2-(4-sulfophenyl)-2H-

tetrazolium], Promega, according to the manufacturer's instructions. The quantity of formazan product was determined by measuring absorbance at $490 \mathrm{~nm}$ using an envision plate reader (PerkinElmer). Flowcytometry was used to determine the percentage of apoptotic cells following treatment with different doses of 8-OHD $(0-70 \mu \mathrm{M})$. The 8-OHD -treated cells were centrifuged $(5 \mathrm{~min}, 1000 \times \mathrm{g})$ at room temperature. Afterward, the cells were resuspended and washed once using $5 \mathrm{ml}$ of phosphate-buffered saline before being stained with annexin-V/PI (apoptosis detection kit; R\&D Systems). Flowcytometry was employed for the analysis according to the manufacturer's protocol.

\section{Real time-PCR}

Total RNA was extracted using the RNeasy mini kit (Qiagen, Germany), according to the manufacturer's instructions. The synthesis of cDNA was performed by reverse transcriptase-polymerase chain reaction (RT-PCR) method using $1 \mu \mathrm{g} / \mathrm{ml}$ total RNA. RNA concentration was measured by Nanodrop 2000c spectrophotometer (Thermo Scientific, USA), and cDNA was synthesized by cDNA Synthesis Kit (Bio FACT, Daejeon, South Korea). The changes in mRNA expression of Wnt1, Notch1, $\beta$-catenin, CXCR4, SOX2, and ALDH3A genes and GAPDH, as internal control, were measured by quantitative reverse transcriptase PCR (qRT-PCR) in a ABI PRISM7900HT (Applied Biosystems) detection system using SYBR GREEN PCR Master Mix. Quantitative RT- PCR was performed using the primers provided in Table.1. 
Table 1. Primer sequences used in this study

\begin{tabular}{lll}
\hline Gene name & Forward primer & Reverse primer \\
\hline Wnt1 & 5'-GAACCTGCTTACAGACTCCAAGAGT-3 & 5'-CCGG ATTTTGG CGTATCAGA-3 \\
Notch1 & 5'-CCGCAGTT GTGCTCCTGAA-3 & 5'-ACCTTGGCGGTCTCGTAGCT-3 \\
B-catenin & 5'-CCTTTGTCCCGCAAATCATG-3 & 5'-ACGTACGGCGCTGGGTATC-3 \\
CXCR4 & 5'-CAGTGGCCGACCTCCTCTT-3 & 5'-ACATGGACTGCCTTGCATAGG -3 \\
SOX2 & 5'-TGCGAGCGCTGCACAT-3 & 5'-CGGGCAGCGTGTACTTATCC-3 \\
ALDH3A1 & 5'-TCCAGCAACGACAAGGTGATT-3 & 5'-GGCAGAGAGTGCAAGGTGATG-3 \\
GAPDH & 5'-ACCCACTCCTCCACCTTTGA-3 & 5'-CT GTTGCTGTAGCCAAATTCGT-3 \\
\hline
\end{tabular}

\section{Western Blot Analysis}

To measure the expression of our tested factors at the protein level, the western blotting technique was used. Firstly, the cells were lysed by using RIPA lysis buffer (Thomas Scientific Inc). A total of 40 $\mu \mathrm{g}$ protein was separated using $10 \%$ SDS-PAGE, electrotransferred onto polyvinylidene fluoride membranes (Millipore, Billerica, MA, USA), blocked with $5 \%$ non-fat milk for $1 \mathrm{~h}$ at room temperature, and incubated with primary antibodies: STAT3, p-STAT3, JAK2, p-JAK2, and anti- $\beta$-actin monoclonal antibodies (Santa Cruz Biotechnology, Santa Cruz, CA, USA) at $4^{\circ} \mathrm{C}$ overnight. Thereafter, the blots were incubated with the corresponding horseradish peroxidase conjugated secondary antibody. Immunoblots were then visualized using an enhanced chemiluminescent kit (SuperSignal; Pie Pierce).

\section{Data Analysis}

Statistical analysis was performed using one-way analysis of variance (ANOVA) followed by Duncan's multiple range test for post hoc comparison by SPSS software version 19. Statistical significance was set at $\mathrm{P}<0.05$ and $\mathrm{P}<0.01$. All the data that were collected from at least three individual experiments were presented as mean $\pm \mathrm{SD}$.

\section{RESULTS}

\section{Enrichment of Breast Cancer Stem-Like Cells by Mammosphere Culture}

Previous studies have evidenced that BCSCs can be propagated in serum-free media supplemented with EGF and bFGF $(24,25)$. Therefore, we employed this cell culture model to enrich the BCSC populations from the MCF-7cell line. $\mathrm{CD} 44^{+} / \mathrm{CD} 24^{-}$ have been previously reported as specific markers for identifying BCSCs (26). After developing mammospheres from MCF-7 cell culture, flowcytometry analysis was performed to sort the breast cancer cell populations based on the expression of CD44 and CD24 in these cells. As depicted in figures $1 \mathrm{~A}$ and $1 \mathrm{~B}$, the expression of $\mathrm{CD} 44^{+} / \mathrm{CD} 24^{-}$cell populations were significantly increased in mammosphere cells. The results also showed that the spheroid culture could be a suitable method to expand the BCSC populations from the MCF-7 cell line. To characterize and confirm the mammosphere cells, the expression levels of stemness markers were quantitatively measured. The results revealed a significant increase in the expression of BCSC stemness markers such as Wnt1, Notch1, $\beta$-catenin, CXCR4, SOX2, and ALDH3A1 in mammosphere culture compared to parental cells (Fig. 1C).

Effects of 8-Hydroxydaidzein on Stemness Markers and Viability of Breast Stem-Like Cells According to previous reports and results of our study, breast cancer cells-derived mammospheres are highly enriched with cells that have BCSC phenotype and generally show increased expression of the BCSC markers (27). In our study, we also examined the effect of 8-OHD $(70 \mu \mathrm{M})$ on stemness markers such as Wnt1, Notch1, $\beta$-catenin, CXCR4, SOX2, and ALDH3A1 in mammosphere cell culture. As shown in Fig.2, 8-OHD treatment significantly reduced the expression of CXCR4, SOX2, and ALDH3A1 in BCSCs- enriched mammospheres. To assess the effect of 8-OHD on the proliferation and viability of cells, mammospheres and parental cells (MCF-7) were treated with varying concentrations of 8-OHD (0$70 \mu \mathrm{M})$ for $48 \mathrm{~h}$. Cell viability was detected by Methyl Tetrazolium salt (MTS) assay. The MTS assay data demonstrated that 8-OHD significantly inhibited the proliferation of mammospheres and parental cells in a dose and time-dependent manner (Fig. 3). According to our obtained data, parental cells were more sensitive to 8-OHD in comparison to the mammosphere cells. 
A
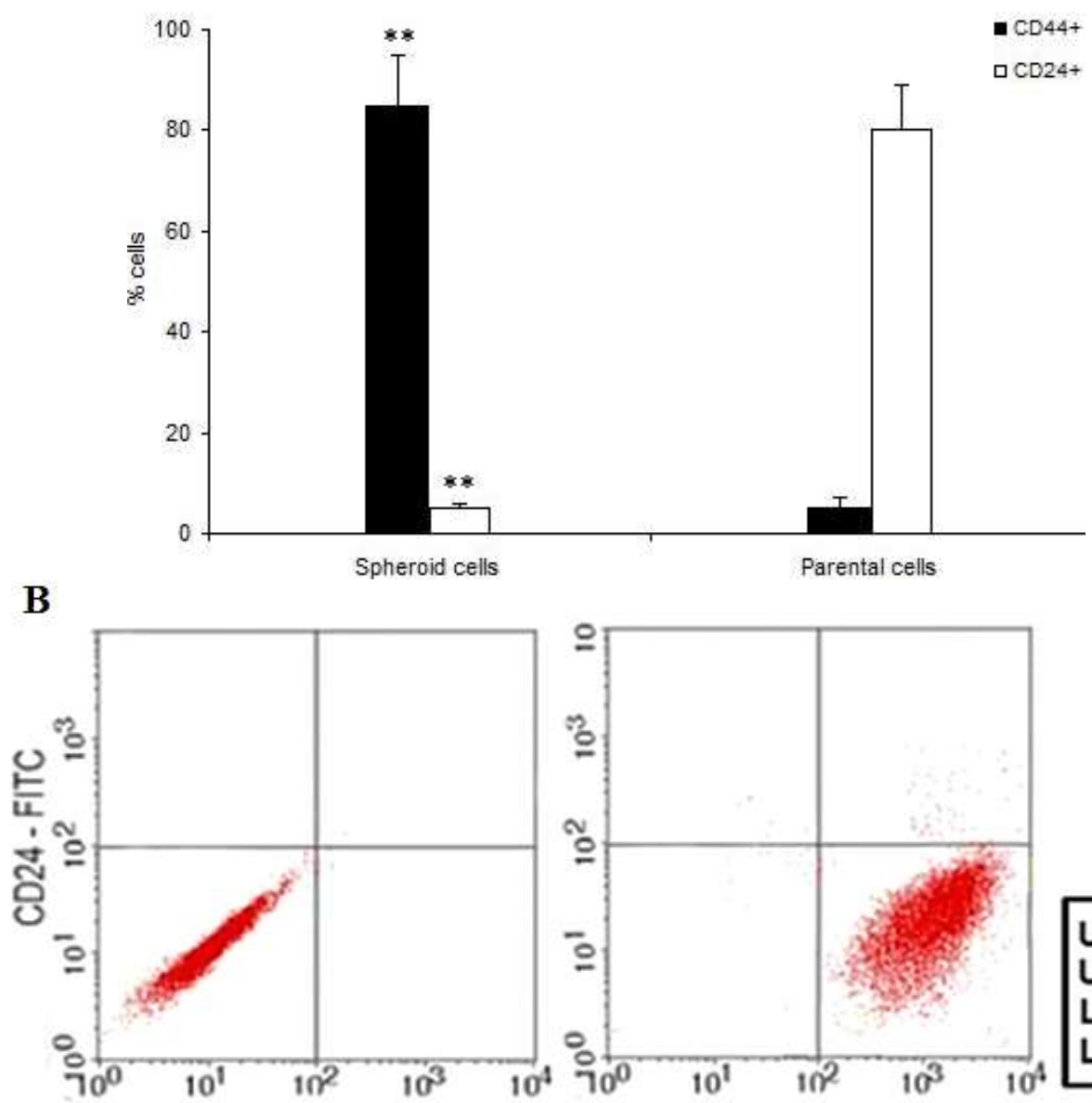

UL: CD44\%CD24*

UR: CD44*/CD24*

LL: CD44/CD24-

LR: $\mathrm{CD} 44^{*} / \mathrm{CD} 24$

C

$\mathrm{CD} 44$ - PE

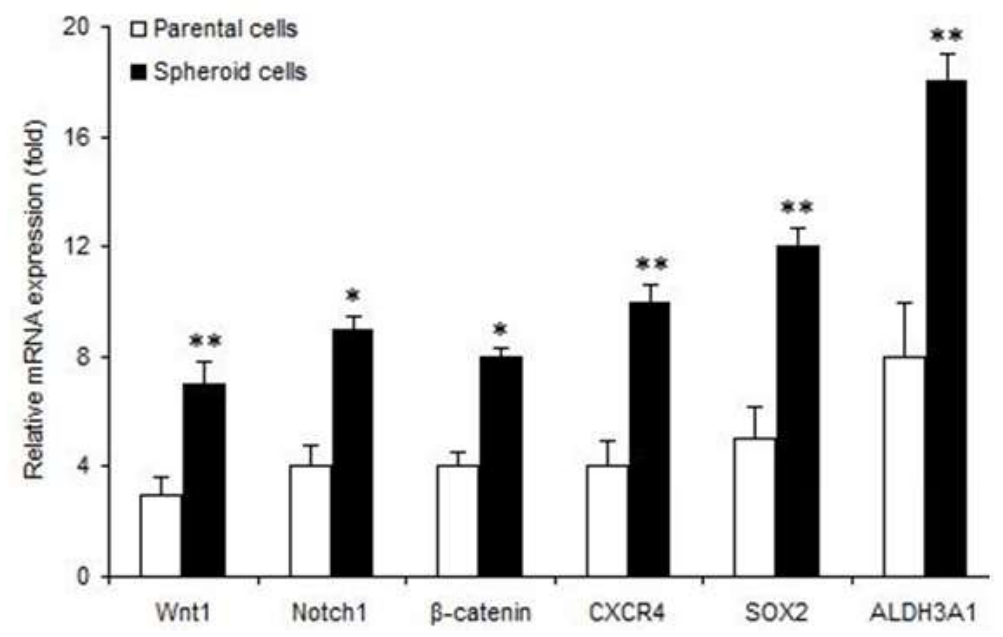

Figure 1. (A) Estimating the percentage of the CD44+/CD24-/low population in MCF-7 cells. Cells were stained with PE$\mathrm{CD} 24$ and FITC-CD44. The results are representative of three separate experiments. Significant differences $(\mathrm{P}<0.05$, Student's t-test) were found between mammosphere cells and parental cells. (B) Flowcytometry analysis data. The fluorescent intensity of CD 24 expression is on the Y axis and of CD44 expression on the X axis. (C) Real-time PCR analysis of the gene expression of Wnt1, Notch1, $\beta$-catenin, CXCR4, SOX2, and ALDH3A1. The data are presented as means $\pm \mathrm{SD}$ for the three separate experiments $(* \mathrm{P}<0.05, * * \mathrm{P}<0.01$, in comparison with the parental cells). 


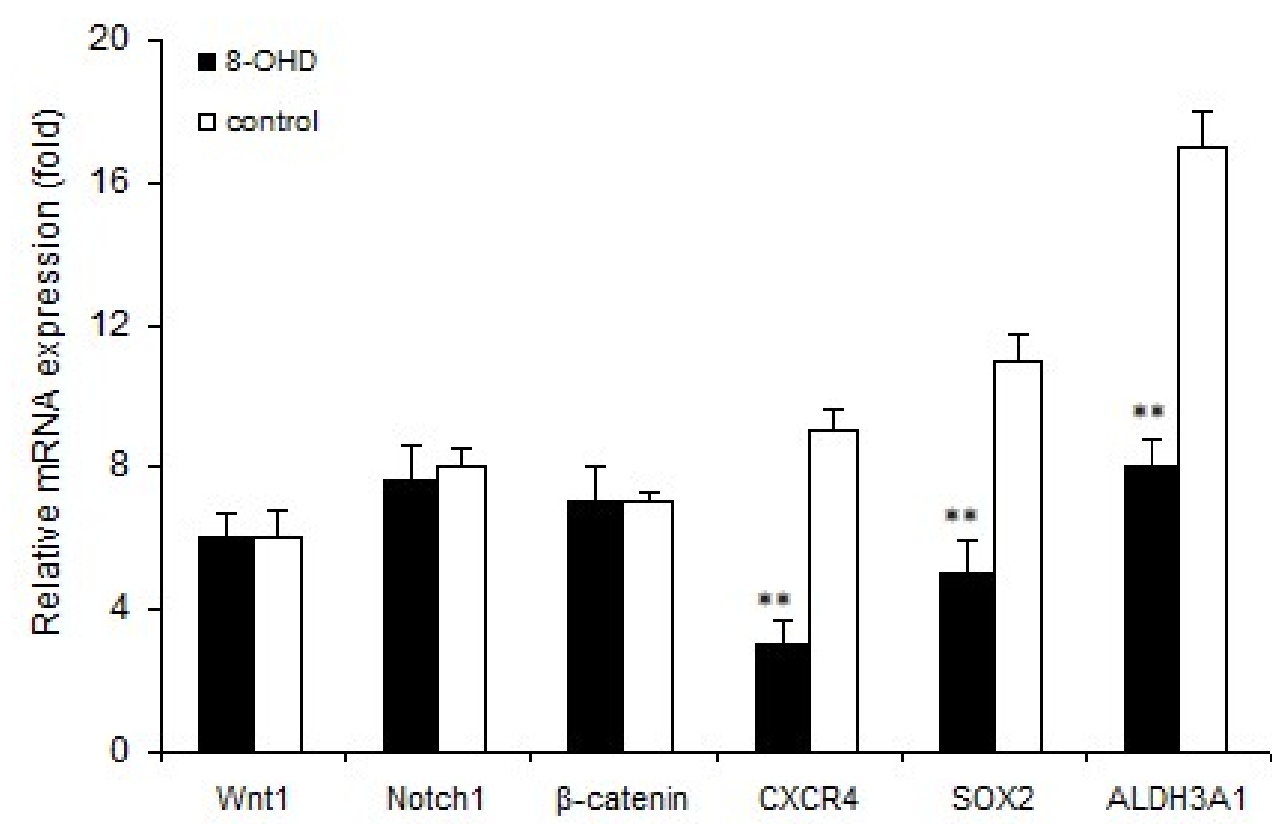

Figure 2. Analysis of the expression levels of stemness markers such as Wnt1, Notch1, $\beta$-catenin, CXCR4, SOX2, and ALDH3A1 in mammosphere cell culture following exposure to $70 \mu \mathrm{M}$ 8-hydroxydaidzein (8-OHD) for 48h. The results are representative of at least three separate experiments. All results were significant at $* * \mathrm{P}<0.01$.

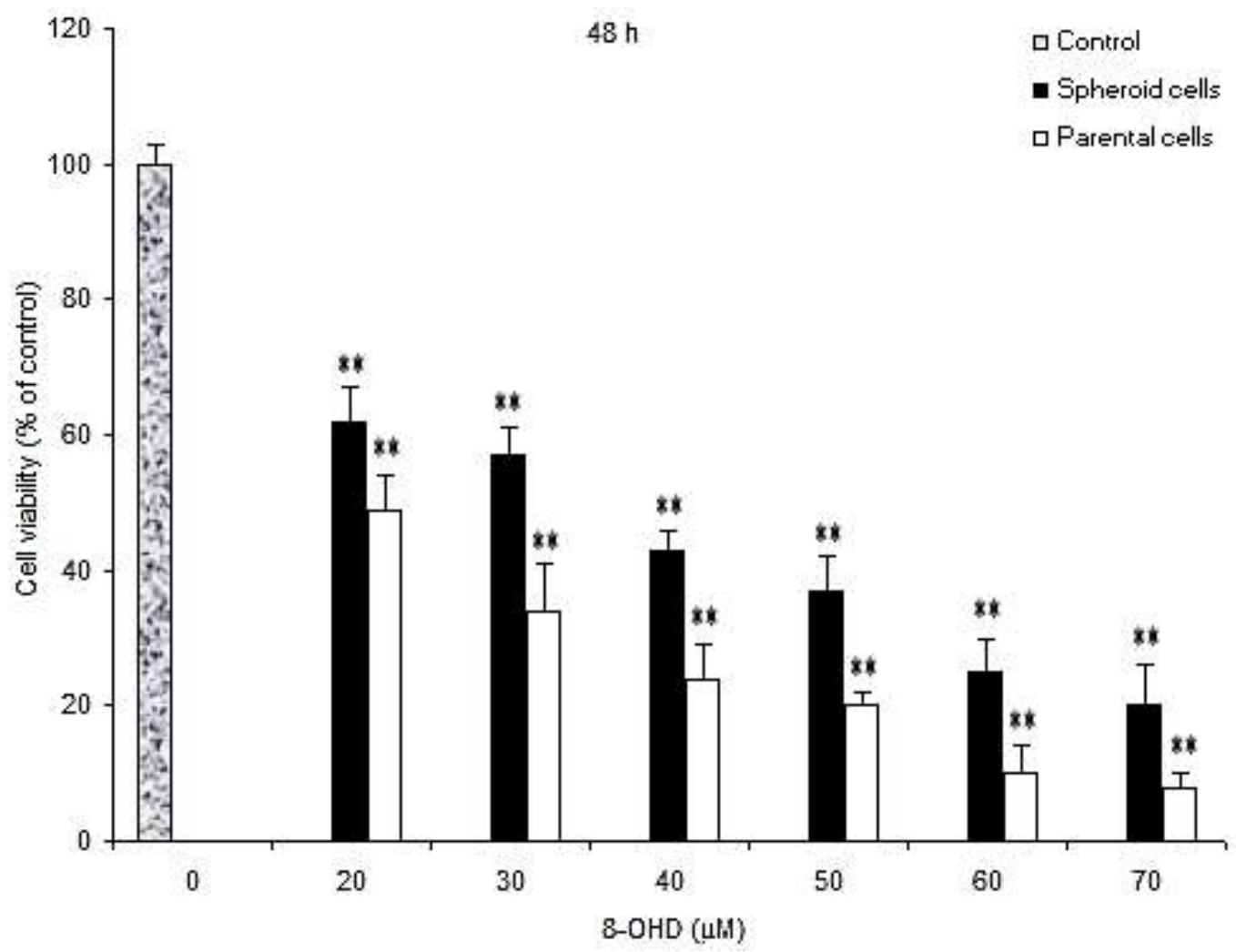

Figure 3. Cytotoxic effects of 8-OHD on MCF-7 spheroid and parental cells. Cells were treated with 8-OHD (0-70 $\mu \mathrm{M})$ for 48 hours. Cell viability was examined using MTS assay. The data are expressed as mean \pm SD of separate experiments. Significant differences $* * \mathrm{P}<0.01$ ) were found between the treatment and control groups. 


\section{Effect of 8-Hydroxydaidzein on Apoptosis in Breast Stem-Like Cells}

As previously has been described, during the early stages of the apoptosis process, phosphatidylserine (PS) translocates to the outer side of the cell membrane. Therefore, we tried to explore the induction of apoptosis by 8-OHD using annexin VFITC/PI staining (28). Accordingly, mammospheres and parental cells were exposed to the different concentrations of 8 -OHD $(0-70 \mu \mathrm{M})$ for 48 hours. The results indicated that 8-OHD markedly induced apoptosis in both mammospheres and parental cells (Fig. 4A). To unravel the involvement of intrinsic or extrinsic pathways in the induction of apoptosis by 8 -OHD, we tended to design additional experiments. According to the literature, fas ligand (FasL or CD95L) plays an important role as death ligand, which mediates apoptosis induction by binding to its receptor, CD95 (29). Besides, anti-Fas (human, neutralizing, clone ZB4) antibody has been identified to block the extrinsic pathway of apoptosis (30). Accordingly, we used CD95L (100 $\mathrm{ng} / \mathrm{ml})$ and $\mathrm{ZB} 4(1 \mu \mathrm{g} / \mathrm{ml}, 1 \mathrm{~h})$ to further elucidate which pathway of apoptosis may be activated by 8 OHD. Flowcytometry analysis of the spheroid and parental cells with either CD95 receptor blocked by pre-incubation with anti-CD95 antibody ZB4 $(1 \mu \mathrm{g} / \mathrm{ml}, 1 \mathrm{~h})$ or unblocked CD95 receptor and subsequent addition of 8 -OHD $(70 \mu \mathrm{M})$ or soluble CD95L $(100 \mathrm{ng} / \mathrm{ml})$ as the positive control for $48 \mathrm{~h}$. The results show that there is a significant difference between the percentages of apoptotic cells in the presence and the absence of ZB4 (Fig. $4 \mathrm{~B}$ and $\mathrm{C}$ ). These data confirmed that 8-OHD induced apoptosis through the intrinsic pathway, suggesting the mechanism of the induction of apoptosis in mammosphere cells. As described in the literature, JAK/STAT signaling pathway has an important role in regulating normal and cancer stem cells behaviors allowing to provoke tumor development (12), and hence the blocking of JAK/STAT signaling pathway leads to the suppression of tumor growth and induction of apoptosis in cancers cells (14). Therefore, we were interested in examining the expression levels of these two proteins in the mammospheres by western blot analysis. The mammospheres were treated with 8 -OHD $(70 \mu \mathrm{M})$ and in combination with interleukin 6 (IL-6) for $48 \mathrm{~h}$. IL- 6 was used to activate cytokine receptors to induce the phosphorylation of STAT3 and JAK2. Treating spheroid cells with IL-6 for 4 hours demonstrated an elevation in the phosphorylation of JAK2 and STAT3 compared to the control group as shown in figure 5. However, 8OHD inhibited the activation of JAK2 and STAT3 in breast stem-like cells by blocking the phosphorylation of these two proteins in comparison to the IL-6 treated group. This compound also apparently prohibited the stimulatory effects of IL- 6 by decreasing the phosphorylation of JAK2 and STAT3 following exposure of the cells to the combination of IL- 6 and 8-OHD.

\section{DISCUSSION}

BCSCs production has been found to play a crucial role in therapy resistance of breast tumors with the ability to regenerate tumor (31). Therefore, targeting these cell populations may aid in improving therapeutic approaches and causes the suppression of tumor growth and progression. Mammosphere culture is one of the efficient ways to isolate and enrichment of tumorigenic breast cancer stem cells (32). Accordingly, we cultured the mammosphere from the MCF-7 cell line to explore the effects of 8-OHD on different aspects of this BCSC-bearing model. To establish the formation of mammospheres enriched with BCSCs, the gene expression levels of a group of stemness markers such as Wnt1, Notch1, $\beta$-catenin, CXCR4, SOX2, and ALDH3A1 as well as CD44/CD24, as BCSC markers, were analyzed. Corresponding data identified high expression levels of all six genes and showed $\mathrm{CD} 44^{+} / \mathrm{CD} 24^{-}$phenotype in mammosphere cells as compared with the parental cells, proposing the formation and enrichment of BCSCs in mammosphere structure. As mentioned earlier, cancer-preventive properties of 8-OHD have been reported in several previous investigations $(16,22)$, However, the precise molecular mechanisms of its anticancer effects, especially against BCSCs, are not yet clear. To meet this purpose, we first examined the effect of this compound on stemness markers in BSCS-enriched mammospheres. Interestingly, we found that 8-OHD significantly reduced the expressions of CXCR4, SOX2, and ALDH3A1 in mammospheres; however, it had no considerable effects on the other three stemness markers in comparison to control cells. To the best of our knowledge, there is no previous data investigating stem cell marker expressions following exposure to daidzein or its derivatives in BCSCs. 


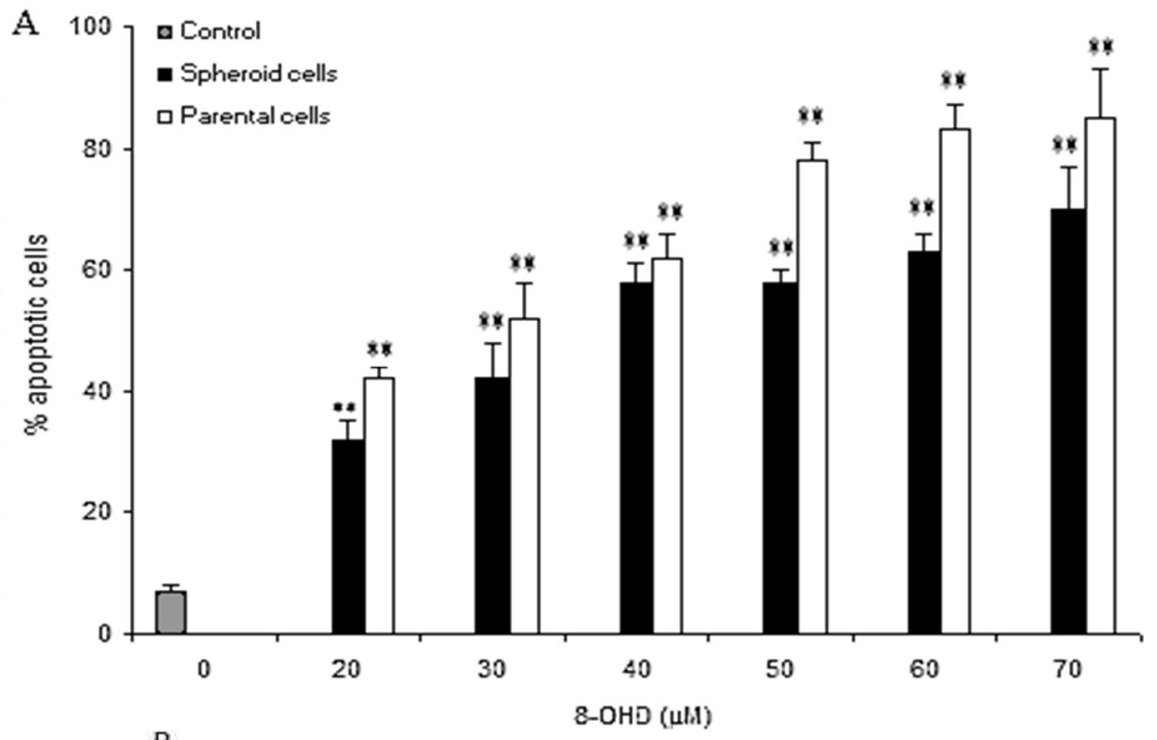

B

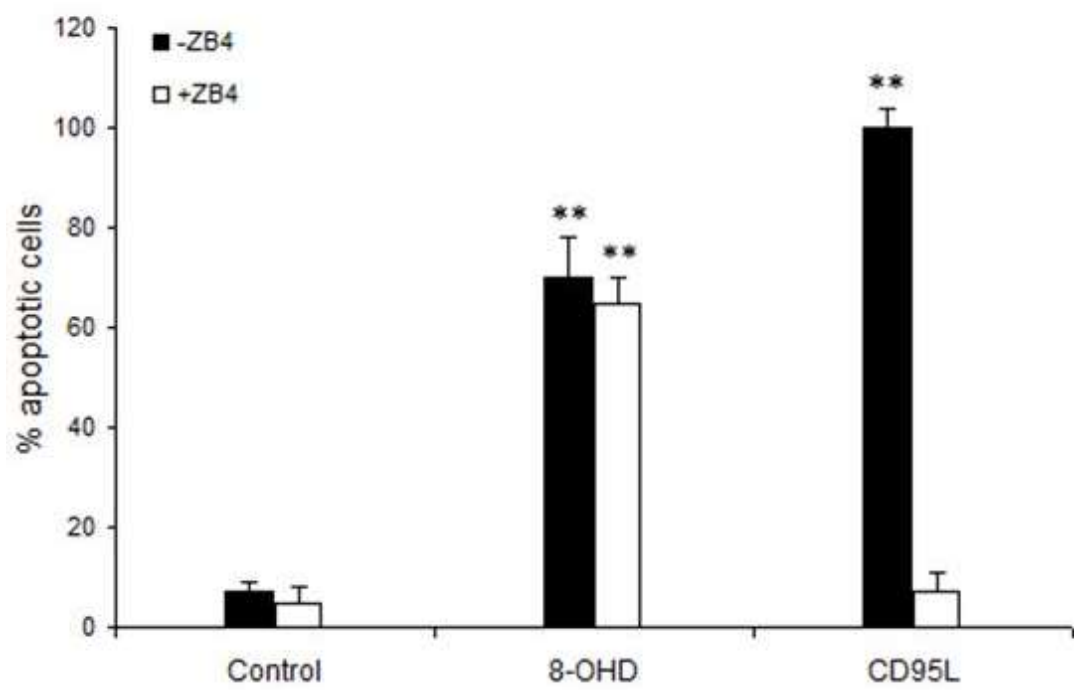



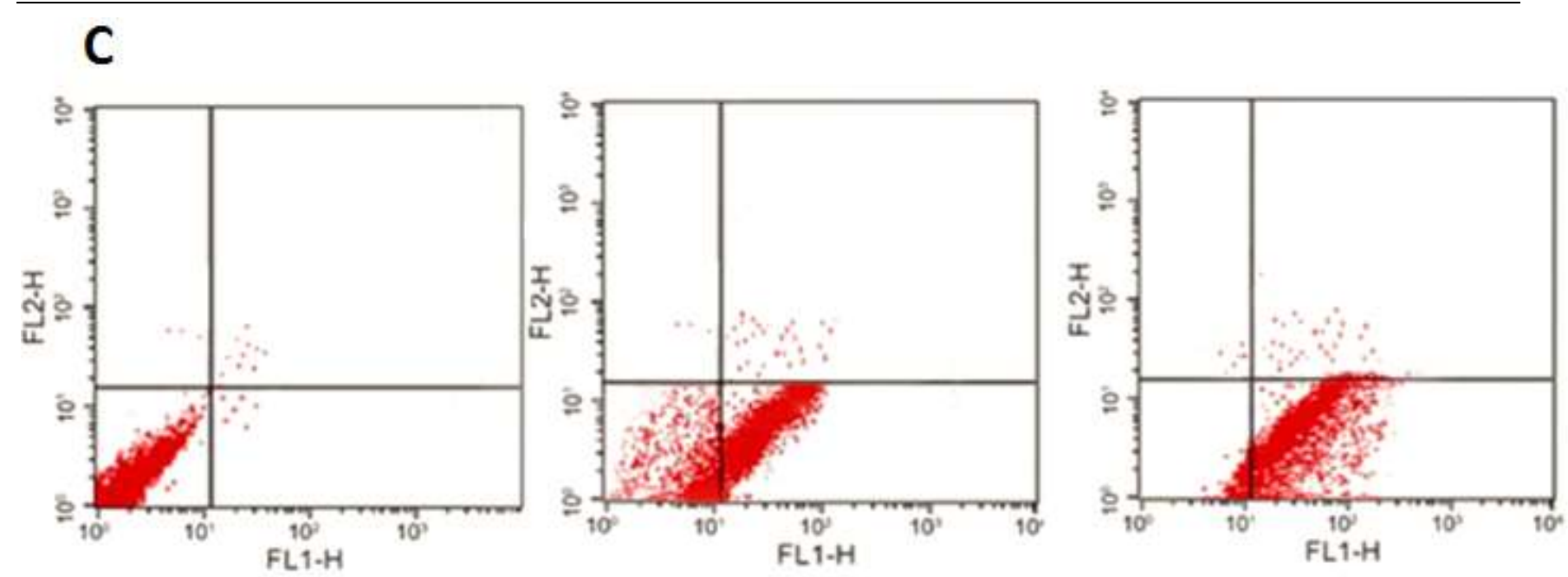

Figure 4. (A) Flowcytometry analysis of the MCF-7 cells treated with 8-Hydroxydaidzein (8-OHD, 0-70 $\mu \mathrm{M})$ for $48 \mathrm{~h}$. (B) 8 -OHD-induced apoptosis occurred independent of CD95L binding to the CD95 receptor $(* * \mathrm{P}<0.01)$. (C) Flowcytometry analysis data.

\section{Control IL-6 8-OHD IL-6+8-OHD}

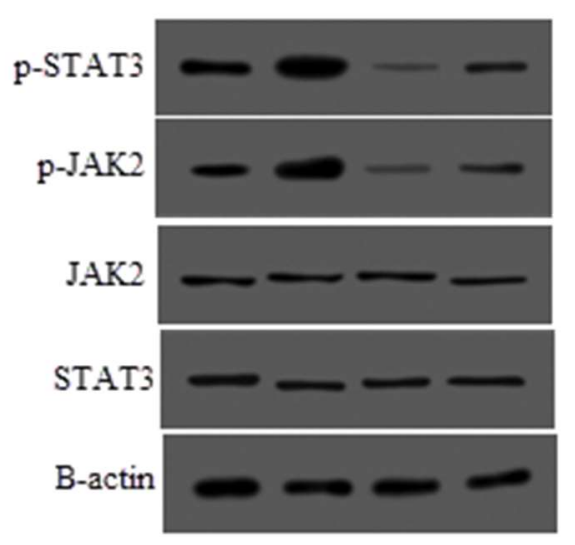

Figure 5. The effects of 8-Hydroxydaidzein (8-OHD, $70 \mu \mathrm{M})$ treatment alone and in combination with interleukin 6 (IL-6, $50 \mathrm{ng} / \mathrm{mL}$ ) on the expressions and phosphorylation of the JAK2 and STAT3 proteins in the spheroid cells. IL-6 was utilized to activate the pathway, leading to the JAK2 and STAT3 phosphorylation. As is illustrated in the figure, 8-OHD inhibited STAT3 and JAK2 phosphorylation induced by IL-6. There are no remarkable differences in the expressions of unphosphorylated proteins between the groups.

However, a study by Kwon, et al. revealed that daidzein had no apparent effect on the expression of stem cell marker, Oct4, in porcine induced Pluripotent Stem Cells (33). They also showed that daidzein didn't affect the apoptosis and proliferation of these stem cells. Another study identified daidzein as a potent ALDH2 inhibitor, the enzyme that has protective effects on the development and differentiation of osteoblastic stem cells (34). The results of that study suggest an inhibitory property for daidzein against this stemness marker, which is in agreement with our observation in the case of ALDH3A1 marker expression. The other study confirmed that daidzein treatment inhibits the formation of ovarian sphere cells, ovospheres, proposing the prohibitory effects of daidzein on stem-like cell properties of these cancer cells (35). The data obtained from that study also identified reduced proliferation, migration, invasion, and induced cell cycle arrest and apoptosis in ovarian cancer cells that seem to occur through regulation of FAK and PI3K/AKT pathway by daidzein, as a phytoestrogen, binding to estrogen receptors. Similarly, our findings showed that the 
treatment of spheroid and parental cells with 8OHD led to a dose-dependent loss of cell viability, and induction of apoptosis in these cells as compared with the control cells. More interestingly, parental cells were more sensitive to 8-OHD in comparison to mammosphere cells. This observation supports the hypothesis that the CSCs are more resistant to the therapeutic agents than the cancer cell itself. Several lines of previous evidence affirmed that daidzein or its derivatives affect cancer cell viability by stimulating the apoptotic process in different types of cancer (36-39). In a second step, we attempted to conduct additional experiments to uncover the mechanism (s) by which 8-OHD may induce cell death. Accordingly, we used CD95L and ZB4 for inducing apoptosis and blocking the extrinsic pathway, respectively. Interestingly, we revealed that 8-OHD stimulates apoptosis via the intrinsic pathway. Some previously published data have also declared that daidzein induces apoptosis of cancer cell lines by modulating the intrinsic pathway $(40,41)$. Additionally, we further examined the effects of 8OHD on JAK/STAT signaling pathway to find a possible molecular mechanism for the intrinsic apoptotic pathway induction. As previously has been unraveled, the inhibition of the JAK2/STAT3 pathway could induce apoptosis via the mitochondrial pathway (42). Therefore, we used IL6 to stimulate the JAK2/STAT pathway and then treated the mammospheres with the combination of IL-6 and 8-OHD as well as the 8-OHD alone. IL-6 considerably increased the levels of phosphorylated JAK2 and STAT3 proteins. However, the 8-OHD treatment decreased the phosphorylation of these proteins either alone or in combination with IL-6 compared with the control and IL-6 treated groups. We proposed that the inhibitory effect of 8-OHD on the phosphorylation of JAK2 and STAT3 may cause the blockage of JAK2/STAT3 signaling and triggers apoptosis of mammosphere cells.

\section{CONCLUSION}

According to our data, we implied that 8-OHD exhibits strong anticancer properties by reducing CSCs characteristics, decreasing cell viability and inducing apoptosis in CSCs and cancer cells. Our study, for the first time, elucidated an anti-BCSC activity for 8-OHD. Moreover, our data suggested a novel mechanism by which 8-OHD may stimulate apoptosis in BCSCs. Taken together; it seems that this daidzein derivative has the potential to be a promising anticancer agent for treating breast cancer. However, we are still very much in the early steps of considering 8-OHD as a potent anti-breast cancer compound, and more detailed experiments are required to prove precise anti-breast cancer mechanisms of this natural product.

\section{ACKNOWLEDGMENT}

We greatly thank the "Shahid Beheshti University of Medical Sciences, Tehran, Iran".

\section{CONFLICT OF INTEREST}

The authors declare that they have no conflict of interest.

\section{REFERENCES}

1. Roco A, Cerda B, Cayun JP, Lavanderos A, Rubilar JC, Cerro R, et al. Pharmacogenetics, tobacco, alcohol and its effect on the risk development cancer. Rev Chil Pediatr. 2018;89(4):432-40. doi: 10.4067/S0370-41062018005000709.

2. Gao Q, Zhou G, Lin SJ, Paus R, Yue Z. How chemotherapy and radiotherapy damage the tissue: Comparative biology lessons from feather and hair models. Exp Dermatol. 2018; 28(4):413-418. doi: 10.1111/exd.13846.

3. Phi LTH, Sari IN, Yang Y-G, Lee S-H, Jun N, Kim $\mathrm{KS}$, et al. Cancer Stem Cells (CSCs) in Drug Resistance and their Therapeutic Implications in Cancer Treatment. Stem Cells Int. 2018. doi: 10.1155/2018/5416923. PubMed PMID: 29681949

4. Dashzeveg NK, Taftaf R, Ramos EK, Torre-Healy L, Chumakova A, Silver DJ, et al. New Advances and Challenges of Targeting Cancer Stem Cells. Cancer Res. 2017;77(19):5222-7. doi: 10.1158/0008-5472.CAN-17-0054.

5. Bray F, Ferlay J, Soerjomataram I, Siegel RL, Torre LA, Jemal A. Global cancer statistics 2018: GLOBOCAN estimates of incidence and mortality worldwide for 36 cancers in 185 countries. CA Cancer J Clin. 2018;68(6):394-424. doi: 10.3322/caac.21492.

6. Lindeman GJ, Visvader JE. Insights into the cell of origin in breast cancer and breast cancer stem cells. Asia Pac J Clin Oncol. 2010;6(2):89-97. doi: 10.1111/j.1743-7563.2010.01279.x.

7. O'Flaherty JD, Barr M, Fennell D, Richard D, Reynolds J, O'Leary J, et al. The cancer stem-cell hypothesis: its emerging role in lung cancer biology and its relevance for future therapy. $\mathrm{J}$ Thorac Oncol. 
2012;7(12):1880-90.

doi: 10.1097/JTO.0b013e31826bfbc6.

8. Zhang F, Song C, Ma Y, Tang L, Xu Y, Wang H. Effect of fibroblasts on breast cancer cell mammosphere formation and regulation of stem cell-related gene expression. Int $\mathrm{J}$ Mol Med. 2011;28(3):365-71. doi: 10.3892/ijmm.2011.700.

9. Zhang F, Xu Y, Song C, Ma Y, Nan F, Wang H, et al. Regulational effects of breast cancer stromal cells and normal breast stromal cells on MCF-7 mammosphere formation. $\mathrm{J}$ Clin Oncol. 2009;27(15S):1056. doi: 10.1200/jco.2009.27.

10. Iqbal J, Chong PY, Tan PH. Breast cancer stem cells: an update. J Clin Pathol. 2013;66(6):485-90. doi: 10.1136/jclinpath-2012-201304.

11. Hermawan A, Putri H. Current report of natural product development against breast cancer stem cells. Int J Biochem Cell Biol. 2018;104:114-32.

12. Galoczova M, Coates P, Vojtesek B. STAT3, stem cells, cancer stem cells and p63. Cell Mol Biol Lett. 2018;23:12. doi: 10.1016/j.biocel.2018.09.012.

13. Liu Y, Gao X, Wang S, Yuan X, Pang Y, Chen J, et al. Cancer Stem Cells are Regulated by STAT3 Signalling in Wilms Tumour. J Cancer. 2018;9(8):1486-99. doi: 10.7150/jca.23277.

14. Xiong H, Zhang ZG, Tian XQ, Sun DF, Liang QC, Zhang YJ, et al. Inhibition of JAK1, 2/STAT3 signaling induces apoptosis, cell cycle arrest, and reduces tumor cell invasion in colorectal cancer cells. Neoplasia. 2008;10(3):287-97. doi: 10.1593/neo.07971.

15. Taylor WF, Jabbarzadeh E. The use of natural products to target cancer stem cells. Am J Cancer Res. 2017;7(7):1588-605. PubMed PMID: 28744407.

16. Kampkotter A, Chovolou Y, Kulawik A, Rohrdanz E, Weber N, Proksch P, et al. Isoflavone daidzein possesses no antioxidant activities in cell-free assays but induces the antioxidant enzyme catalase. Nutr Res. 2008;28(9):620-8. doi: 10.1016/j.nutres.2008.06.002.

17. Seo MH, Kim BN, Kim KR, Lee KW, Lee CH, Oh DK. Production of 8-hydroxydaidzein from soybean extract by Aspergillus oryzae KACC 40247. Biosci Biotechnol Biochem. 2013;77(6):1245-50. doi: 10.1271/bbb.120899.

18. Kulling SE, Honig DM, Simat TJ, Metzler M. Oxidative in vitro metabolism of the soy phytoestrogens daidzein and genistein. J Agric Food Chem. 2000;48(10):4963-72. doi: 10.1021/jf000524i.

19. Wu PS, Ding HY, Yen JH, Chen SF, Lee KH, Wu MJ. Anti-inflammatory Activity of 8Hydroxydaidzein in LPS-Stimulated BV2 Microglial Cells via Activation of Nrf2-Antioxidant and Attenuation of Akt/NF-kappaB-Inflammatory Signaling Pathways, as Well As Inhibition of COX-
2 Activity. J Agric Food Chem. 2018;66(23):5790801. doi: $10.1021 /$ acs.jafc. 8 b00437.

20. Rimbach G, De Pascual-Teresa S, Ewins BA, Matsugo S, Uchida Y, Minihane AM, et al. Antioxidant and free radical scavenging activity of isoflavone metabolites. Xenobiotica. 2003;33(9):913-25. doi: 10.1080/0049825031000150444.

21. Rufer CE, Kulling SE. Antioxidant activity of isoflavones and their major metabolites using different in vitro assays. J Agric Food Chem. 2006;54(8):2926-31. doi: 10.1021/jf053112o.

22. Lo YL. A potential daidzein derivative enhances cytotoxicity of epirubicin on human colon adenocarcinoma Caco-2 cells. Int $\mathrm{J}$ Mol Sci. 2012;14(1):158-76. doi: 10.3390/ijms14010158.

23. Lombardo Y, de Giorgio A, Coombes CR, Stebbing $\mathrm{J}$, Castellano L. Mammosphere formation assay from human breast cancer tissues and cell lines. J Vis Exp. 2015(97). doi: 10.3791/52671.

24. Phillips TM, McBride WH, Pajonk F. The response of CD24(-/low)/CD44+ breast cancer-initiating cells to radiation. J Natl Cancer Inst. 2006;98(24):177785. doi: $10.1093 /$ jnci/djj495.

25. Huang MZ, Zhang FC, Zhang YY. Influence factors on the formation of mammospheres from breast cancer stem cells. Beijing Da Xue Xue Bao Yi Xue Ban. 2008;40(5):500-4.

26. Horimoto Y, Arakawa A, Sasahara N, Tanabe M, Sai S, Himuro T, et al. Combination of Cancer Stem Cell Markers CD44 and CD24 Is Superior to ALDH1 as a Prognostic Indicator in Breast Cancer Patients with Distant Metastases. PLoS One. 2016;11(10):e0165253. doi: 10.1371/journal.pone.0165253.

27. Stivarou T, Stellas D, Vartzi G, Thomaidou D, Patsavoudi E. Targeting highly expressed extracellular HSP90 in breast cancer stem cells inhibits tumor growth in vitro and in vivo. Cancer Biol Ther. 2016;17(8):799-812. doi: 10.1080/15384047.2016.1195041.

28. Thomas E, Gopalakrishnan V, Somasagara RR, Choudhary B, Raghavan SC. Extract of Vernonia condensata, Inhibits Tumor Progression and Improves Survival of Tumor-allograft Bearing Mouse. Sci Rep. 2016;6:23255. doi: 10.1038/srep23255.

29. Peter ME, Hadji A, Murmann AE, Brockway S, Putzbach W, Pattanayak A, et al. The role of CD95 and CD95 ligand in cancer. Cell Death Differ. 2015;22(5):885-6. doi: 10.1038/cdd.2015.25.

30. Shi LS, Wang H, Wang F, Feng M, Wang M, Guan WX. Effects of gastrokine2 expression on gastric cancer cell apoptosis by activation of extrinsic apoptotic pathways. Mol Med Rep. 2014;10(6):2898-904. doi: 10.3892/mmr.2014.2603. 
31. Zhang Y, Xu B, Zhang XP. Effects of miRNAs on functions of breast cancer stem cells and treatment of breast cancer. Onco Targets Ther. 2018;11:426370. doi: 10.2147/OTT.S165156.

32. Grimshaw MJ, Cooper L, Papazisis K, Coleman JA, Bohnenkamp HR, Chiapero-Stanke L, et al. Mammosphere culture of metastatic breast cancer cells enriches for tumorigenic breast cancer cells. Breast Cancer Res. 2008;10(3):R52. doi: $10.1186 / \mathrm{bcr} 2106$.

33. Kwon DJ, Hwang IS, Kwak TU, Yang H, Park MR, Ock SA, et al. Effects of Cell Cycle Regulators on the Cell Cycle Synchronization of Porcine induced Pluripotent Stem Cells. Dev Reprod. 2017;21(1):4754. doi: 10.12717/DR.2017.21.1.047.

34. Chen CH, Ferreira JC, Gross ER, Mochly-Rosen D. Targeting aldehyde dehydrogenase 2: new therapeutic opportunities. Physiol Rev. 2014;94(1):1-34. doi: 10.1152/physrev.00017.2013.

35. Chan KKL, Siu MKY, Jiang YX, Wang JJ, Leung THY, Ngan HYS. Estrogen receptor modulators genistein, daidzein and ERB-041 inhibit cell migration, invasion, proliferation and sphere formation via modulation of FAK and PI3K/AKT signaling in ovarian cancer. Cancer Cell Int. 2018;18:65. doi: 10.1186/s12935-018-0559-2.

36. Park HJ, Jeon YK, You DH, Nam MJ. Daidzein causes cytochrome c-mediated apoptosis via the Bcl2 family in human hepatic cancer cells. Food Chem Toxicol. 2013;60:542-9. doi: 10.1016/j.fct.2013.08.022.

37. Choi EJ, Kim GH. Daidzein causes cell cycle arrest at the G1 and G2/M phases in human breast cancer
MCF-7 and MDA-MB-453 cells. Phytomedicine. 2008;15(9):683-90. 10.1016/j.phymed.2008.04.006.

38. Somjen D, Grafi-Cohen M, Katzburg S, Weisinger G, Izkhakov E, Nevo N, et al. Anti-thyroid cancer properties of a novel isoflavone derivative, 7-(O)carboxymethyl daidzein conjugated to N-t-Bochexylenediamine in vitro and in vivo. J Steroid Biochem Mol Biol. 2011;126(3-5):95-103. doi: 10.1016/j.jsbmb.2011.04.009.

39. Green JM, Alvero AB, Kohen F, Mor G. 7-(O)Carboxymethyl daidzein conjugated to N-t-Bochexylenediamine: a novel compound capable of inducing cell death in epithelial ovarian cancer stem cells. Cancer Biol Ther. 2009;8(18):1747-53. doi: 10.4161/cbt.8.18.9285.

40. Tang S, Hu J, Meng Q, Dong X, Wang K, Qi Y, et al. Daidzein induced apoptosis via down-regulation of $\mathrm{Bcl}-2 / \mathrm{Bax}$ and triggering of the mitochondrial pathway in BGC-823 cells. Cell Biochem Biophys. 2013;65(2):197-202. doi: 10.1007/s12013-0129418-2.

41. Jin S, Zhang QY, Kang XM, Wang JX, Zhao WH. Daidzein induces MCF-7 breast cancer cell apoptosis via the mitochondrial pathway. Ann Oncol. 2010;21(2):263-8. doi: 10.1093/annonc/mdp499.

42. Du W, Hong J, Wang YC, Zhang YJ, Wang P, Su WY, et al. Inhibition of JAK2/STAT3 signalling induces colorectal cancer cell apoptosis via mitochondrial pathway. J Cell Mol Med. 2012;16(8):1878-88. doi: $10.1111 / j .1582-$ 4934.2011.01483.x. 